DESY-09-071 , MPP-2009-58

\title{
Constraining resonant photon-axion conversions in the Early Universe
}

\author{
Alessandro Mirizzi \\ Max-Planck-Institut für Physik (Werner Heisenberg Institut) \\ Föhringer Ring 6, 80805 München, Germany \\ Javier Redondo \\ Deutsches Elektronen Synchrotron \\ Notkestraße 85, 22607 Hamburg, Germany
}

Günter Sigl

II. Institut für theoretische Physik, Universität Hamburg, Luruper Chaussee 149, 22761 Hamburg, Germany

\begin{abstract}
The presence of a primordial magnetic field would have induced resonant conversions between photons and axion-like particles (ALPs) during the thermal history of the Universe. These conversions would have distorted the blackbody spectrum of the cosmic microwave background (CMB). In this context, we derive bounds on the photon-ALP resonant conversions using the high precision CMB spectral data collected by the FIRAS instrument on board of the Cosmic Background Explorer. We obtain upper limits on the product of the photon-ALP coupling constant $g$ times the magnetic field strength $B$ down to $g B \lesssim 10^{-13} \mathrm{GeV}^{-1} \mathrm{nG}$ for ALP masses below the eV scale.

Keywords: axions, cosmic microwave background
\end{abstract}




\section{Introduction}

The possible existence of a primordial magnetic field of cosmological origin has been the subject of an intense investigation during the last few decades. Despite these efforts, no astrophysical evidence has been reported so far concerning magnetic fields over cosmological scales, and only upper limits are reported. When scaling the original bound from the Faraday effect of distant radio sources $[1,2]$ to the now much better known baryon density measured by the Wilkinson Microwave Anisotropy Probe (WMAP) [3], one has $B \lesssim 2.8 \times 10^{-7}\left(l_{c} / \mathrm{Mpc}\right)^{-1 / 2} \mathrm{G}$, coherent on a scale $l_{c} \simeq 1 \mathrm{Mpc}$ [4]. A recent analysis of the WMAP 5-year data on the Faraday rotation of the linear polarization of the cosmic microwave background $(\mathrm{CMB})$ gives comparable upper limits ranging from $6 \times 10^{-8}$ to $2 \times 10^{-6} \mathrm{G}[5]$.

The presence of a primordial magnetic field would inevitably produce resonant conversions between photons and axion-like particles (ALPs) in the Early Universe. This effect was pointed out at first in a work by Yanagida and Yoshimura [6]. They realized that such a conversion could produce a sizable distortion in the CMB spectrum. Since at that time data indicated a distortion in the Wien region of the CMB, these authors speculated that a resonant axion-photon conversion could be an intriguing explanation for such an effect. Nowadays, the blackbody nature of the CMB spectrum has been measured with a precision better than 1 part in $10^{4}$ by the Far Infrared Absolute Spectrophotometer (FIRAS) on board of the Cosmic Background Explorer (COBE) $[7,8]$. The astonishing accuracy of such a measurement now allows to constrain exotic scenarios which would deplete the CMB spectrum, such as hidden photons [9, 10], axions [11], radiative neutrino decays [12] or millicharged particles [13]. Recent bounds on photon-ALP mixing using the precision CMB data [11] have focused only on nonresonant conversions in the late Universe. However, for values of ALP masses allowing resonant conversions during the expansion of the Universe one expects stronger bounds due to this resonant enhancement. For this reason, in the present paper we determine the bounds from resonant photon-ALP conversions in light of the COBE precision CMB data. Moreover, we make use of an accurate description of the evolution of the cosmological plasma in which the resonant conversions arise, following the line of our recent study on resonant photon-hidden photon conversions in the Early Universe [10].

Often, strong bounds on the photon-ALP coupling arise from stellar evolution [14]. Our bounds are difficult to compare with these other constraints since they strongly depend on the strength of the primordial fields. If fields near the experimental bounds $\sim 100 \mathrm{nG}$ are realized, our bounds can be much stronger than stellar evolution constraints, but they could also be weaker if the primordial fields were much smaller. However, in general, our bounds are complementary to stellar evolution ones, since some ALP models [15-19] predict a suppression of the photon-ALP coupling in dense stellar interiors, which would not happen in the relatively diluted primordial plasma. For this reason, we think worthwhile to explore which independent constraints are achievable from cosmological arguments. 
The plan of our work is as follows. In Section 2 we review the mechanism of mixing between photons and axion-like particles and we present our analytical prescription to calculate the resonant photon-ALP conversion probability in the expanding Universe. In Section 3 we describe our simplified model for the effective photon mass induced by the primordial plasma. In Section 4 we discuss the effect of the random primordial magnetic fields on the resonant conversions and we characterize the averaging of the conversion probability over the sky and over the photon polarization, relevant to study ALP effects on the CMB monopole spectrum. In Section 5 we describe the constraints coming from spectral CMB distortions for ALP masses undergoing resonant conversions after the recombination epoch. In Section 6 we extend our limits to pre-recombination resonances, using the experimental limits on the chemical potential $\mu$ of the CMB spectrum and the agreement between Big Bang Nucleosynthesis (BBN) and the CMB on the effective number of additional relativistic species at decoupling. Finally, in Section 7 we comment on the complementarity of our bound with other astrophysical and experimental constraints and present our conclusions.

\section{Photon mixing with axion-like particles}

\subsection{Axion-like Particles}

Axion-like particles (ALPs) are scalar or pseudoscalar bosons $\phi$ that couple to two photons with one of the following interaction Lagrangians [20]

$$
\begin{aligned}
& \mathcal{L}_{\text {pseudoscalar }}=-\frac{1}{4} g_{-} F_{\mu \nu} \widetilde{F}^{\mu \nu} \phi=g_{-} \mathbf{B} \cdot \mathbf{E} \phi, \\
& \mathcal{L}_{\text {scalar }}=\frac{1}{4} g_{+} F_{\mu \nu} F^{\mu \nu} \phi=\frac{1}{2} g_{+}\left(\mathbf{B}^{2}-\mathbf{E}^{2}\right) \phi,
\end{aligned}
$$

where $\widetilde{F}^{\mu \nu}=\epsilon^{\mu \nu \alpha \beta} F_{\alpha \beta} / 2$ is the dual of the field strength tensor and $\mathbf{E}$ and $\mathbf{B}$ are the electric and magnetic fields, respectively. In the presence of a constant external magnetic field one can decompose the electromagnetic field into an external component and the dynamical part representing photons as $\mathbf{B} \rightarrow \mathbf{B}^{\text {ext }}+\nabla \times \mathbf{A}$. The terms in the Lagrangian containing $\mathbf{B}^{\text {ext }}$ act as mass mixing terms between the axion-like particle $\phi$ and the photon field $A_{\mu}=\left(A_{0} ; \mathbf{A}\right)$. Choosing the radiation gauge $\nabla \cdot \mathbf{A}=0$ the mixing terms become evident,

$$
\begin{array}{ll}
\mathcal{L}_{\text {pseudoscalar }} & =g_{-} \mathbf{B}^{\text {ext }} \cdot \partial_{0} \mathbf{A} \phi+\ldots, \\
\mathcal{L}_{\text {scalar }} & =g_{+} \mathbf{B}^{\text {ext }} \cdot(\nabla \times \mathbf{A}) \phi+\ldots .
\end{array}
$$

In our study we will consider the mixing of photons with these particles. If we write A as a plane wave with frequency $\omega$ and wave vector $\mathbf{k}$ we can explicitly evaluate its time and spatial derivatives. Reabsorbing a factor of $i$ into $\mathbf{A}$ and using $\omega \simeq|\mathbf{k}|$ we find that the mixing part in the Lagrangian reads

$$
\begin{array}{ll}
\mathcal{L}_{\text {pseudoscalar }}^{\text {mix }} & =g_{-} B_{T} \omega A_{\|} \phi, \\
\mathcal{L}_{\text {scalar }}^{\text {mix }} & =g_{+} B_{T} \omega A_{\perp} \phi,
\end{array}
$$


where $B_{T}$ is the component of the external magnetic field perpendicular to the propagation direction of photons $(\mathbf{k})$ and $A_{\|}, A_{\perp}$ are respectively the components of A parallel and perpendicular to that component. Note that the component of $\mathbf{B}^{\text {ext }}$ parallel to $\mathbf{k}$ does not contribute to the mixing and also that pseudoscalar fields mix with the parallel component $A_{\|}$, while scalars mix with $A_{\perp}$. In the following, we denote with $g$ the ALP-photon coupling constant and consider only the photon component, $\gamma$, that mixes with $\phi$.

Due to the effective mass-mixing in the external magnetic field, the propagating eigenstates in vacuum are now rotated with respect to $(\gamma, \phi)$ by an angle $\theta$ given by [21]

$$
\begin{aligned}
& \sin 2 \theta=\frac{2 g B \omega}{\sqrt{m_{\phi}^{4}+(2 g B \omega)^{2}}}, \\
& \cos 2 \theta=\frac{m_{\phi}^{2}}{\sqrt{m_{\phi}^{4}+(2 g B \omega)^{2}}},
\end{aligned}
$$

where, for simplicity, we have indicated the transverse component of the magnetic field with $B$, and $m_{\phi}$ is the ALP mass. This misalignment is well known to produce $\gamma \leftrightarrow \phi$ oscillations with a wavenumber given by [21]

$$
k=\frac{\sqrt{m_{\phi}^{4}+(2 g B \omega)^{2}}}{2 \omega} \equiv \frac{\Delta m^{2}}{2 \omega} .
$$

\subsection{Medium effects and resonant $M S W$ conversions}

Photon oscillations into ALPs are modified by the refractive properties of the medium. In the primordial plasma, photons acquire a non-trivial dispersion relation which can be parametrized by adding an effective photon mass $m_{\gamma}$ to the Lagrangian. This is generally complex, reflecting the absorption properties of the plasma. However, for most of the parameter space studied here it turns out that absorption is negligible. Moreover, even when this is not the case our results will show no dependence on it. Therefore, we have chosen to neglect it in the following exposition and include a few remarks when relevant. In this case, the effective mixing angle $\tilde{\theta}$ is related to the vacuum one by [14]

$$
\begin{aligned}
& \sin 2 \tilde{\theta}=\frac{\sin 2 \theta}{\left[\sin ^{2} 2 \theta+(\cos 2 \theta-\xi)^{2}\right]^{1 / 2}}, \\
& \cos 2 \tilde{\theta}=\frac{\cos 2 \theta-\xi}{\left[\sin ^{2} 2 \theta+(\cos 2 \theta-\xi)^{2}\right]^{1 / 2}},
\end{aligned}
$$

where the parameter $\xi$ which measures the significance of the medium effects reads

$$
\xi=\frac{m_{\gamma}^{2}}{\sqrt{m_{\phi}^{4}+(2 g B \omega)^{2}}}=\cos 2 \theta\left(\frac{m_{\gamma}}{m_{\phi}}\right)^{2} .
$$

As the Universe expands, eventually the condition

$$
m_{\phi}=m_{\gamma}
$$


is satisfied and $\tilde{\theta} \rightarrow \pi / 4$. When this condition is fulfilled, resonant photon-ALP conversions are possible, analogous to the well-known Mikheev-Smirnov-Wolfenstein (MSW) effect for neutrino flavor transitions [22-24]. If the photon production and detection points are separated by many oscillation lengths from a resonance, the oscillation patterns wash out. Thus, the transition probability is given by [25]

$$
P_{\gamma \rightarrow \phi} \simeq \frac{1}{2}+\left(p-\frac{1}{2}\right) \cos 2 \theta_{0} \cos 2 \tilde{\theta}
$$

where we have assumed the mixing angle $\theta_{0}$ at the detection in vacuum, $\tilde{\theta}$ is the effective mixing angle at the production point (considered to be at high density) and $p$ is the level crossing probability. This latter takes into account the deviation from adiabaticity of photon-ALP conversions in the resonance region. In particular, one has $p=0$ for a completely adiabatic transition and $p=1$ for an extremely nonadiabatic one. The crossing probability $p$ for photon-ALP resonant conversions can be obtained using the Landau-Zener expression [10]

$$
p \simeq \exp \left(-2 \pi r k \sin ^{2} \theta_{r}\right),
$$

where $k$ is again the $\gamma \rightarrow \phi$ vacuum oscillation wavenumber in Eq. (9), $\theta_{r}$ is the vacuum mixing angle at the resonance and

$$
r=\left|\frac{d \ln m_{\gamma}^{2}(t)}{d t}\right|_{t=t_{\mathrm{res}}}^{-1}
$$

is a scale parameter to be evaluated at the location where a resonance occurs. $\ddagger$

Note that the mixing angle $\theta$ changes as the Universe evolves because the frequency $\omega$ and the magnetic field $B$ are functions of the redshift. Denoting with $\omega_{0}$ and $B_{0}$ their values at $z=0$, the frequency grows as $\omega=\omega_{0}(1+z)$ while the magnetic field evolution is model-dependent. In this paper we have decided to focus in the most widely studied case of considering magnetic fields frozen into the medium, for which $B=B_{0}(1+z)^{2}[2]$. It is clear from Eq. (7) that at early times the vacuum mixing angle becomes maximal, $\sin 2 \theta \rightarrow 1$. However, the effective mixing angle $\tilde{\theta}$ remains small because of the suppression by matter effects. In fact, at early times, before recombination, $m_{\gamma}^{2}$ is given by the plasma frequency $\omega_{\mathrm{P}}^{2}$ which is proportional to the free electron density which scales as $(1+z)^{3}$. Therefore, at high redshifts $(z \rightarrow \infty)$, $\xi$ tends indeed to a constant given by

$$
\xi \rightarrow \frac{m_{\gamma}^{2}}{2 g B \omega}=\frac{m_{\gamma}^{2}(z=0)}{2 g B_{0} \omega_{0}} \simeq 2.7 \times 10^{5}\left(g_{10} B_{0, \mathrm{nG}}\right)^{-1}\left(\frac{T_{0}}{\omega_{0}}\right),
$$

where we have used $m_{\gamma}(z=0)=1.59 \times 10^{-14} \mathrm{eV}[10]$, normalized the photon frequency today $\omega_{0}$ with respect to the actual $\mathrm{CMB}$ temperature $T_{0}=2.725 \mathrm{~K}[8]$ and finally defined $g_{10}=g / 10^{-10} \mathrm{GeV}^{-1}$ and $B_{0, \mathrm{nG}}=B_{0} / 1 \mathrm{nG}$ as typical values close to their

$\ddagger$ Our problem is different to the widely discussed of neutrino oscillations or the photon-hidden photon case studied in [10] since here even the vacuum mixing evolves in time. However, the corrections to Eq. (15) that we are neglecting are unimportant, since the evolution of the vacuum mixing $\theta$ is very smooth near the resonance, contrarily to the evolution of $\tilde{\theta}$. 
current experimental limits. For such large value of $\xi$ we can take $\cos 2 \tilde{\theta} \simeq-1$ at the production point in Eq. (14).

Moreover, the FIRAS sensitivity will allow us to bound $P_{\gamma \rightarrow \phi} \lesssim 10^{-4}$ which also excludes non-resonant vacuum oscillations with $\sin ^{2} 2 \theta_{0} \gtrsim 10^{-4}$. Considering resonant conversions we will constrain much smaller mixings so that we can also take $\cos 2 \theta_{0} \simeq 1$ in Eq. (14). This then simplifies to

$$
P_{\gamma \rightarrow \phi} \simeq 1-p
$$

The FIRAS bounds thus require a strongly non-adiabatic resonance. Under this condition, we can approximate the conversion probability as

$$
P_{\gamma \rightarrow \phi} \simeq 2 \pi r k \sin ^{2} \theta_{r}
$$

The product $r k$ is basically the ratio of the characteristic expansion time of the universe and the vacuum $\gamma \rightarrow \phi$ oscillation length, and for the region of parameters considered here is large. Since FIRAS constrains $P_{\gamma \rightarrow \phi} \lesssim 10^{-4}$, this tells us that the vacuum mixing angle $\theta_{r}$ at the resonance has to be extremely small. Therefore, we will work in the small mixing regime, for which $\Delta m^{2} \simeq m_{\phi}^{2}$ and

$$
\sin 2 \theta_{r} \simeq 0.92 \times 10^{-5}(1+z)^{3}\left(g_{10} B_{0, \mathrm{nG}}\right)\left(\frac{\omega_{0}}{T_{0}}\right)\left(\frac{10^{-14} \mathrm{eV}}{m_{\phi}}\right)^{2},
$$

where in Eq. (7) we have approximated $\theta_{r} \simeq g B \omega / m_{\phi}^{2}$, since at the resonance $m_{\phi}=m_{\gamma}$ and $m_{\phi}^{2} \gg 2 g B \omega$ for the values of ALP masses we are considering. Under, this approximation, the expression for the conversion probability is exceedingly simple, i.e.

$$
P_{\gamma \rightarrow \phi} \simeq \frac{g^{2} B^{2} \pi r \omega}{m_{\phi}^{2}}
$$

\section{Cosmological $m_{\gamma}$ profile}

In order to calculate the conversion probability we need the profile of the photon effective mass $m_{\gamma}$ along the cosmological line of sight. In this work we use the same prescription of our Ref. [10] in which we studied the resonant conversions between photons and hidden photons in the expanding Universe.

The effective mass can be parametrized as

$$
m_{\gamma}^{2}=\omega_{\mathrm{P}}^{2}\left(X_{e}\right) \times\left[1-0.0073\left(\frac{\omega}{\mathrm{eV}}\right)^{2}\left(\frac{1-X_{e}}{X_{e}}\right)\right],
$$

where $\omega_{\mathrm{P}}\left(X_{e}\right) \simeq 1.59 \times 10^{-14}(1+z)^{3 / 2} X_{e}^{1 / 2} \mathrm{eV}$ is the plasma frequency with $X_{e}(z)$ the hydrogen ionization fraction as a function of redshift, which we take from [27]. We note that the CMB energies probed by FIRAS are much smaller than the first excitation energies of the hydrogen (when the temperatures are low enough to allow bound atoms) so that we have neglected these effects in the expression of the effective mass. We have also ignored the subleading helium contribution. Based on [10], in Fig. 1 we show our reference profile for the cosmological history of the effective photon mass $m_{\gamma}$ as a 


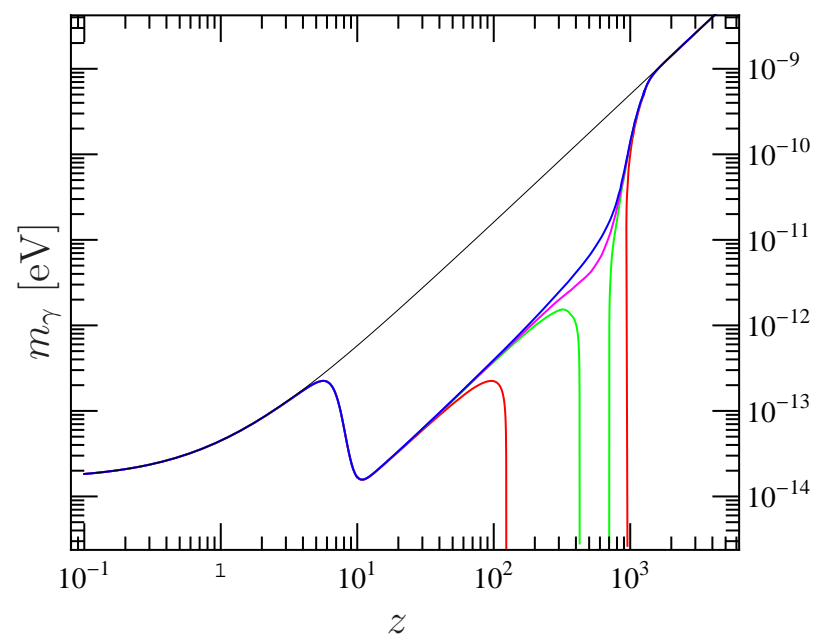

Figure 1. Effective photon mass as function of redshift. The thin line uses $X_{e}=1$, the blue, magenta, green and red lines are for $\omega / T=1,3,4,10$, respectively. These values are in the frequency range probed by FIRAS. The two sharp dips in the green and red lines bound the region where $m_{\gamma}^{2}$ becomes negative. (See [10] for further details)

function of redshift $z$. We recall that the evolution of the effective photon mass which depends on the history of the ionization fraction $X_{e}(z)$ and also on the photon energy, is extremely complex. Above a temperature $T \sim 0.5 \mathrm{eV}$ (redshift $z \sim 1100$ ) hydrogen is fully ionized. As the Universe temperature decreases, photons cannot ionize hydrogen efficiently and electrons and protons slowly combine. This makes the Universe very transparent to radiation, indeed releasing the photon bath which we see today as the CMB. This is the so-called recombination epoch. Later on $(z \lesssim 6)$ the Universe becomes ionized again due to ultraviolet radiation from the first quasars or population III stars. These effects produce the non-monotonic behavior in the shape of the effective photon mass as a function of redshift seen in Fig. 1. Note also that during recombination the effective mass squared can become negative if the photon energy is large enough.

Along such a non-monotonic mass profile multiple resonances can occur for a given ALP mass (provided $m_{\phi} \lesssim 10^{-12} \mathrm{eV}$ ). If this is the case, Eq. (21) tells us that the most adiabatic crossing, quantitatively the more important, is the one that happens at earlier times. This is particularly clear when we express all red-shift dependent quantities as their values at $z=0$ times their redshift dependence and use $r \propto(3 H)^{-1}$, with $H$ the Hubble constant, to find $P_{\gamma \rightarrow \phi} \propto(1+z)^{5} / H$ which increases with redshift since $H$ grows at most as $(1+z)^{2}$ during the radiation dominated epoch.

We note that for photon energies $\omega / T \gtrsim 3.8$ there is a small period during the dark ages for which the effective mass squared becomes negative. This implies that there is one redshift at which $m_{\gamma}^{2}=0$ and at which, in principle, ALPs of arbitrarily small mass can be resonantly produced. However, due to the fast drop of $m_{\gamma}^{2}$, this crossing is extremely non-adiabatic, as we have explicitly checked. For this reason, we will not consider this case hereafter. 
In summary, in the case of multiple crossings the most relevant one happens before reionization. As a consequence, for $m_{\phi} \gtrsim 10^{-14} \mathrm{eV}$ we need to evaluate resonances only for $z \gtrsim 10$. This situation simplifies our calculations because after reionization the plasma is complicated by the presence of density inhomogeneities [10].

\section{Magnetic fields at the resonance and probability averages}

In principle, the primordial magnetic field is not known, although it is generally expected to show a somewhat turbulent structure [28]. We would like to clarify how it affects the resonant photon-ALP conversions. For this reason, we have to compare the typical width of a resonance region with the coherence scale of the magnetic field. The halfwidth of the resonance is, according to Eq. $(10), \delta \xi(t)=\sin 2 \theta_{r}$, which corresponds to a length scale

$$
\begin{aligned}
\tau_{r} & =r \sin 2 \theta_{r} \\
& \lesssim 1.3 \times 10^{-2}(1+z)^{3 / 2}\left(g_{10} B_{0, \mathrm{nG}}\right)\left(\frac{\omega_{0}}{T_{0}}\right)\left(\frac{10^{-14} \mathrm{eV}}{m_{\phi}}\right)^{2} \mathrm{Mpc} \\
& \lesssim 0.42\left(g_{10} B_{0, \mathrm{nG}}\right)\left(\frac{\omega_{0}}{T_{0}}\right)\left(\frac{10^{-14} \mathrm{eV}}{m_{\phi}}\right) \mathrm{Mpc},
\end{aligned}
$$

where for the first numerical estimate we have used Eq. (20) and $r \lesssim(3 H)^{-1} \simeq 1.4(1+$ $z)^{-3 / 2}$ Gpc, with $H$ the Hubble constant. Eq. (24) results from the fact that for the most relevant resonance before reionization (at $z \gtrsim 10)$ one has $m_{\phi}=m_{\gamma} \gtrsim 10^{-14}[(1+$ $z) / 10]^{3 / 2} \mathrm{eV}$. The resonance half-width is thus smaller than the coherence length $l_{c} \sim$ $l_{c, 0}(1+z)^{-1}$ of the magnetic field for

$$
g_{10} B_{0, \mathrm{nG}} \lesssim 1.3 \times 10^{-2}\left(\frac{m_{\phi}}{10^{-14} \mathrm{eV}}\right)^{1 / 3}\left(\frac{T_{0}}{\omega_{0}}\right)\left(\frac{l_{c, 0}}{\mathrm{Mpc}}\right) .
$$

The boundary of our exclusion bounds will satisfy this constraint (see Fig. 3) so that we can consider the magnetic field constant during the resonance to compute it. At couplings larger than the ones at the exclusion boundary, the resonance width will eventually become larger than the magnetic field coherence length and photons will see different magnetic field domains during the resonance. Nevertheless, the transition probability increases by increasing the value of $g$, so that we can also exclude this upper region. Therefore, also in such a situation, we will continue to use our simple prescription of taking the magnetic field evaluated exactly at the crossing point, defined by our Eq. (13), and constant during the resonance§.

However, the magnetic field direction and strength during the resonance will generally be different along different directions in the sky. Moreover, the photon-ALP resonant conversions depend on the relative orientation of the photon polarization and the magnetic field direction, so that in different magnetic domains different photon

$\S$ Possible corrections due to the variation of $B$ inside of the resonant region (see, e.g., the discussion in [26] in the neutrino case) are not expected to modify our exclusion plot above the boundary. 


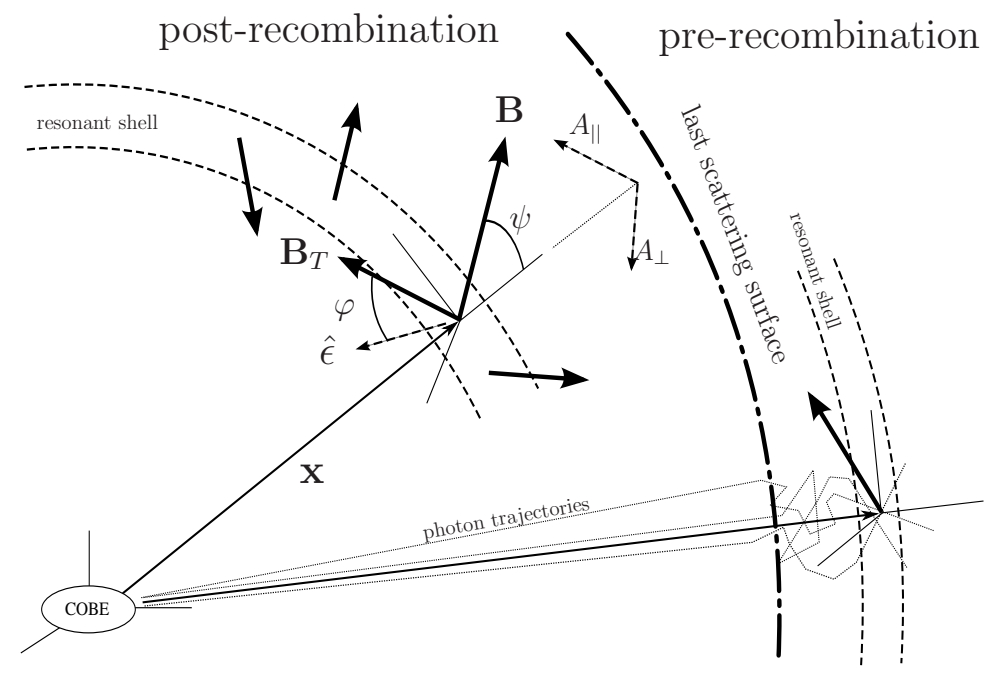

Figure 2. Picture of resonant photon-ALP oscillations of the cosmic microwave radiation. The transitions happen during a very short time in the history of the universe (the resonant shell) determined by the ALP mass which here is shown confined by dashed lines. The primordial magnetic field can be assumed to be constant during the resonance (the shell thickness) but both its strength and orientation depend on the angular position in the sky. Resonant transitions happening after recombination deplete CMB photons depending on their polarization. If resonances happen before recombination, photon trajectories can form any angle with $\mathbf{B}$ during the resonance and then be rescattered towards the observer such that information about the polarization is lost. In this case, the CMB light from the last scattering surface is effectively averaged over the angle $\psi$.

polarization states play the role of $A_{\|}$and $A_{\perp}$, see Fig. 2. For a generic photon polarization, the $B$ strength entering the conversion probability in Eq. (21) is\|

$$
B=|\mathbf{B}(\mathbf{x}) \cdot \hat{\epsilon}|=|\mathbf{B}(\mathbf{x}) \sin \psi(\mathbf{x}) \cos \varphi|,
$$

where $\mathbf{x}$ is the position vector of the resonance region in a particular direction $\hat{x}, \hat{\epsilon}$ is the photon polarization vector $(|\hat{\epsilon}|=1, \hat{\epsilon} \times \hat{x}=0), \psi(\mathbf{x})$ is the angle between the magnetic field $\mathbf{B}(\mathbf{x})$ and the photon propagation direction $\hat{x}$ and $\varphi$ the angle between $\mathbf{B}_{T}$ (the component of the magnetic field perpendicular to $\hat{x}$ ) and $\hat{\epsilon}$, see Fig. 2.

In this paper we will mainly discuss bounds of photon-ALP mixing from the CMB monopole. If the resonance happens after recombination, the full-sky and polarization averaged photon-ALP conversion probability Eq. (21) is

$$
\left\langle P_{\gamma \rightarrow \phi}\right\rangle=\frac{\pi g^{2} r \omega}{m_{\phi}^{2}} \frac{1}{3}\left\langle B^{2}\right\rangle,
$$

|| Eq. (26) holds for parity-odd ALPs; when considering parity-even one finds $|\mathbf{B}(\mathbf{x}) \times \hat{\epsilon}|=$ $|\mathbf{B}(\mathbf{x}) \sin \psi(\mathbf{x}) \sin \varphi|$ instead. 
where we have defined a conveniently normalized average

$$
\begin{aligned}
\left\langle B^{2}\right\rangle & \equiv 3 \int \frac{d \Omega_{\hat{x}}}{4 \pi} \int_{0}^{2 \pi} \frac{d \varphi}{2 \pi}|\mathbf{B}|^{2} \sin ^{2} \psi \cos ^{2} \varphi \\
& =\frac{3}{2} \int \frac{d \Omega_{\hat{x}}}{4 \pi}|\mathbf{B}|^{2} \sin ^{2} \psi,
\end{aligned}
$$

when we recall that implicitly $\mathbf{B}$ and $\psi$ depend on $\mathbf{x}$. The convenience of this average is clarified in two simple cases: a) the absolute value $|\mathbf{B}|$ is constant in the whole sky but has a random direction and b) it has a fixed direction. In both cases we find

$$
\left\langle B^{2}\right\rangle=|\mathbf{B}|^{2} .
$$

When the resonance happens before recombination, the photon trajectories can form any angle $\psi$ with $\mathbf{B}$ during the resonance and then be re-scattered towards the observer such that information about the polarization is lost. Since the photon trajectories are not straight, the $\psi$ angle is not correlated with the magnetic field direction, photon polarization and the direction in the sky. Because of this, one has to perform a local average over $\psi$ in each magnetic domain before doing the sky-average, i.e.

$$
|\mathbf{B}|^{2} \int \frac{d \varphi}{2 \pi} \frac{d \Omega_{\psi}}{4 \pi} \sin ^{2} \psi \cos ^{2} \varphi=\frac{1}{3}|\mathbf{B}|^{2}
$$

Assuming that $\mathbf{B}=\mathbf{B}(\mathbf{x})$ does not vary very much on the distance scales of the horizon at last-scattering surface, we shall then define a sky average

$$
\left\langle B^{2}\right\rangle=\int \frac{d \Omega_{\hat{x}}}{4 \pi}|\mathbf{B}|^{2}
$$

so that also in the pre-recombination epoch one can use Eq. (27) for the conversion probability. Note that if $|\mathbf{B}|$ is isotropic, also in this case we obtain $\left\langle B^{2}\right\rangle=|\mathbf{B}|^{2}$. This expression neglects in principle decoherence effects from photon scattering absorption during the resonance. However as we will see in Sec. 6 these effects are not relevant if the conversion probability is small.

\section{Bounds in the post-recombination era}

The CMB spectrum measured by FIRAS fits extremely well to a black-body spectrum at a temperature $T_{0}=2.725 \pm 0.002 \mathrm{~K}$ [8]. The energy range of the CMB spectrum measured by FIRAS [7] is $2.84 \times 10^{-4} \mathrm{eV} \leq \omega_{0} \leq 2.65 \times 10^{-3} \mathrm{eV}$, corresponding to $1.2 \leq \omega_{0} / T_{0} \leq 11.3$. In that region, the $\mathrm{CMB}$ blackbody becomes unprotected to distortions below a cosmic temperature $\sim 50 \mathrm{eV}$, which corresponds to a photon plasma mass of $\sim 10^{-6} \mathrm{eV}$. On the other hand, today the average plasma mass for photons $m_{\gamma}$ is as low as $2 \times 10^{-14} \mathrm{eV}$. If ALPs exist with a mass between these two values they will be produced resonantly and leave their imprint on the CMB as a frequency-dependent distortion [see Eq. (27)]. Bounds for higher masses are considered in the following section.

In order to obtain our bound, we have considered the distortion of the overall blackbody spectrum. To this end we use the COBE-FIRAS data for the experimentally 
measured spectrum, corrected for foregrounds [7]. Note that the new calibration of FIRAS [8] is within the old errors and would not change any of our conclusions. The $N=43$ data points $\Phi_{i}^{\exp }$ at different frequencies $\omega_{i}$ are obtained by summing the best-fit blackbody spectrum to the residuals reported in Ref. [7]. The errors $\sigma_{i}^{\exp }$ are also available. In the presence of photon-ALP conversion, the original intensity of the "theoretical blackbody" monopole at temperature $T$,

$$
\Phi^{0}(\omega, T)=\frac{\omega^{3}}{\pi^{2}}[\exp (\omega / T)-1]^{-1}
$$

would be deformed to

$$
\Phi(\omega, T, \lambda)=\Phi^{0}(\omega, T)\left[1-\left\langle P_{\gamma \rightarrow \phi}\right\rangle\right],
$$

where $\left\langle P_{\gamma \rightarrow \phi}\right\rangle$ is the sky average of the polarization averaged photon-ALP conversion probability, defined in Eq. (27). We can then build the reduced chi-squared function

$$
\chi_{\nu}^{2}(T, \lambda)=\frac{1}{N-1} \sum_{i}^{N}\left[\frac{\Phi_{i}^{\exp }-\Phi\left(\omega_{i}, T, \lambda\right)}{\sigma_{i}^{\exp }}\right]^{2} .
$$

We minimize this function with respect to $T$ for each point in the parameter space $\lambda=\left(m_{\phi}, g\left\langle B^{2}\right\rangle^{1 / 2}\right)$, i.e. $T$ is an empirical parameter determined by the $\chi_{\nu}^{2}$ minimization for each $\lambda$ rather than being fixed at the standard value.

In Fig. 3 we show our exclusion contour. In particular, the region above the continuous curve is the excluded region at 95\% C.L., i.e. in this region the chance probability to experimentally obtain larger values of $\chi_{\nu}^{2}$ is lower than $5 \%$.

We shall stress again that the CMB photons from different angles have traversed magnetic fields during the resonance that have in principle different strengths and directions. This would induce anisotropies and polarisation. The difference between a direction parallel and orthogonal to the magnetic field must not exceed the observed CMB temperature anisotropy, $\Delta T / T<10^{-5}$. However, the bound achievable from this anisotropy pattern is expected to be less stringent than the one put from the distortion of the overall blackbody spectrum [11]. Possible improvement of this bound would require a detailed investigation in terms of the multipole expansion of the CMB temperature fluctuations. We deserve this task for a future work.

\section{Bounds in the pre-recombination era}

The resonant conversion of photons into ALPs produces an energy-dependent depletion of the CMB which can be constrained by FIRAS data. However, if this resonance happens before recombination, the CMB is still coupled to the primordial plasma and these distortions can be processed by photon-plasma interactions which will tend to thermalize the spectrum. Photon scattering and absorption during the resonance can in principle affect our result Eq. (21) producing a damping of the photon-ALP conversions. Both effects can be introduced in an imaginary contribution to the effective photon mass squared. In the limit of strong damping, the flavor relaxation rate becomes independent on the details of the scattering processes. When this situation dominates a resonance, 
taking the results of [9] (computed in the photon-hidden photon case but valid for a general mixing case) and translating them into our problem in the relevant small mixing case, we obtain

$$
P_{\gamma \rightarrow \phi} \simeq \frac{1}{2}\left(1-p^{2}\right)=\frac{1}{2}\left[1-\exp \left(-2 \frac{\pi g^{2} B^{2} \omega}{3 H m_{\phi}^{2}}\right)\right],
$$

where $p$ is the same crossing probability given by Eq. (15). The effects of decoherence become manifest in the fact that for adiabatic resonances, $p \sim 0$, the system reaches thermal equilibrium, and photons and ALPs equilibrate their populations which, of course, results in a transition probability of $1 / 2$. However, in the non-adiabatic limit, this expression gives exactly our Eq. (21), with $r=(3 H)^{-1}$ in this regime, so our results do not depend on whether the resonance is vacuum or damping dominated and we can use Eq. (21) in both the cases. For the sake of the simplicity, we neglect possible cases in which the scales of the damping and of the resonance are of the same order. As a summary, we only have to consider the processing of the CMB spectrum after the resonance to compare it with the FIRAS data.

Note that the processing strongly depends on the temperature at which the resonance takes place, since it determines if the different photon interactions are effective or not. The response of the primordial plasma to distortions of the Planck distribution is driven by two fundamental classes of processes: Compton scattering conserves the number of photons, whereas double Compton scattering, Bremsstrahlung and their time reversal analogues change the number of photons.

Compton scattering is the fastest process and is responsible for the change of photon direction and polarization described already in Sec. 4. Compton scattering can also be effective at redistributing the energies of photons, however per se cannot change the photon number. It can provide kinetic equilibrium of the photon distribution provided it is efficient, i.e.,

$$
\int_{0}^{z} \frac{n_{e} \sigma_{\mathrm{Th}}}{2 H} \frac{T}{m_{e}} \frac{d z}{1+z} \gg 1 \quad \rightarrow \quad z \gg 2 \times 10^{5}
$$

where $\sigma_{\mathrm{Th}}$ is the Thompson's scattering cross section. This condition corresponds to masses $m_{\phi} \gg 1.4 \times 10^{-6} \mathrm{eV}$. Therefore, below this mass the plasma is so weakly coupled that it cannot process the distortion. In such a condition, the treatment given in the previous section holds with the only difference being the averaging of photon directions during the resonance, as discussed in Sec. 4. The corresponding bound is shown in Fig. 3.

For masses $\sim 10^{-6} \mathrm{eV}$ the situation is more complex since the resonance happens when Compton scattering is neither efficient nor inefficient. The evolution of the distortions has to be studied by numerically evolving the spectrum with the Kompaneets equation. We believe this is beyond the scope of this paper.

Whenever Compton scattering is efficient, the photon distribution acquires, in short time scale after the resonance, a Bose-Einstein shape characterized by a chemical 
potential. For small distortions, this is given by [29]

$$
\mu_{r}=\frac{-1}{2.142}\left(3 \frac{\delta \rho}{\rho}-4 \frac{\delta n}{n}\right) \simeq-0.05 g_{10}^{2}\left\langle B_{0, \mathrm{nG}}^{2}\right\rangle,
$$

where $\delta \rho / \rho$ and $\delta n / n$ are the fractions of the CMB energy and photon number converted into ALPs during the resonance. Note that, in contrast to the case often studied in the literature, resonant photon-ALP conversion absorbs relatively more energy than photon number and therefore produces negative chemical potentials.

Inverse Double Compton scattering (DC) and Bremsstrahlung (BS) occur much less frequent than Compton scattering but in contrast to the latter can change the photon number. Therefore, on a longer time scale they can absorb the necessary photons to recreate a pure Planck distribution, i.e. they can erase the chemical potential. For small distortions, the evolution of $\mu$ can be approximated by [29]

$$
\begin{aligned}
& \frac{d \mu}{d t}=-\mu\left(\frac{1}{t_{\mathrm{DC}}}+\frac{1}{t_{\mathrm{BS}}}\right), \quad \text { with } \\
& t_{\mathrm{DC}}=1.06 \times 10^{8} z_{6}{ }^{9 / 2} \mathrm{~s} . \quad ; \quad t_{\mathrm{BS}}=3.73 \times 10^{8} z_{6}{ }^{13 / 4} \mathrm{~s},
\end{aligned}
$$

where we have taken the values $Y_{p}=0.25, \Omega_{b} h^{2}=0.0223$ for the $\Lambda$ CDM model and defined $z_{6}=z / 10^{6}$. During radiation domination we can write the expansion time as $t=(2 H)^{-1} \simeq 2.38 \times 10^{7} z_{6}^{-2} \mathrm{~s}$. We have computed for each ALP mass the time at which the resonance takes place and the evolution of the chemical potential. The FIRAS data sets the bound on the value of the chemical potential today $\mu_{0}<9 \times 10^{-5}$, which gives the exclusion range denoted $\mu$ in Fig. 3 .

The bounds from the distortions of the CMB spectrum vanish very fast for masses above $\sim 0.1 \mathrm{meV}$ because Double Compton scattering and Bremsstrahlung become very efficient. However, there is a further bound we can consider for resonances happening in the post BBN epoch. During ALP production a fraction of the energy stored in the CMB is transferred to ALPs, which immediately decouple from the thermal bath. If the ALPs produced in this way have sufficiently small masses to be relativistic during the epoch of the CMB formation (i.e. roughly $m_{\phi} \lesssim 1 \mathrm{eV}$ ) then they behave as a nonstandard contribution to the radiation energy density. Therefore, the radiation energy density measured from CMB anisotropies would keep track of the ALPs contribution. A comparison of the radiation energy density during Big Bang Nucleosynthesis (not affected by ALPs resonant production) and CMB decoupling gives an upper limit on this contribution, namely $x<\delta \rho / \rho=0.2[9,30]$. In our case this translates into a bound

$$
g_{10}\left\langle B_{0, \mathrm{nG}}^{2}\right\rangle^{1 / 2}<1.4
$$

We show our combined constraints in Fig. 3. In this figure we have used the full expression in Eq. (35) since above $m_{\phi} \simeq 0.1 \mathrm{meV}$ the photon-ALP conversion probability bound cannot be considered small (also, for any mass a fraction of 0.2 of the photons converted into ALPs requires $P_{\gamma \rightarrow \phi}$ of order 1 ). 


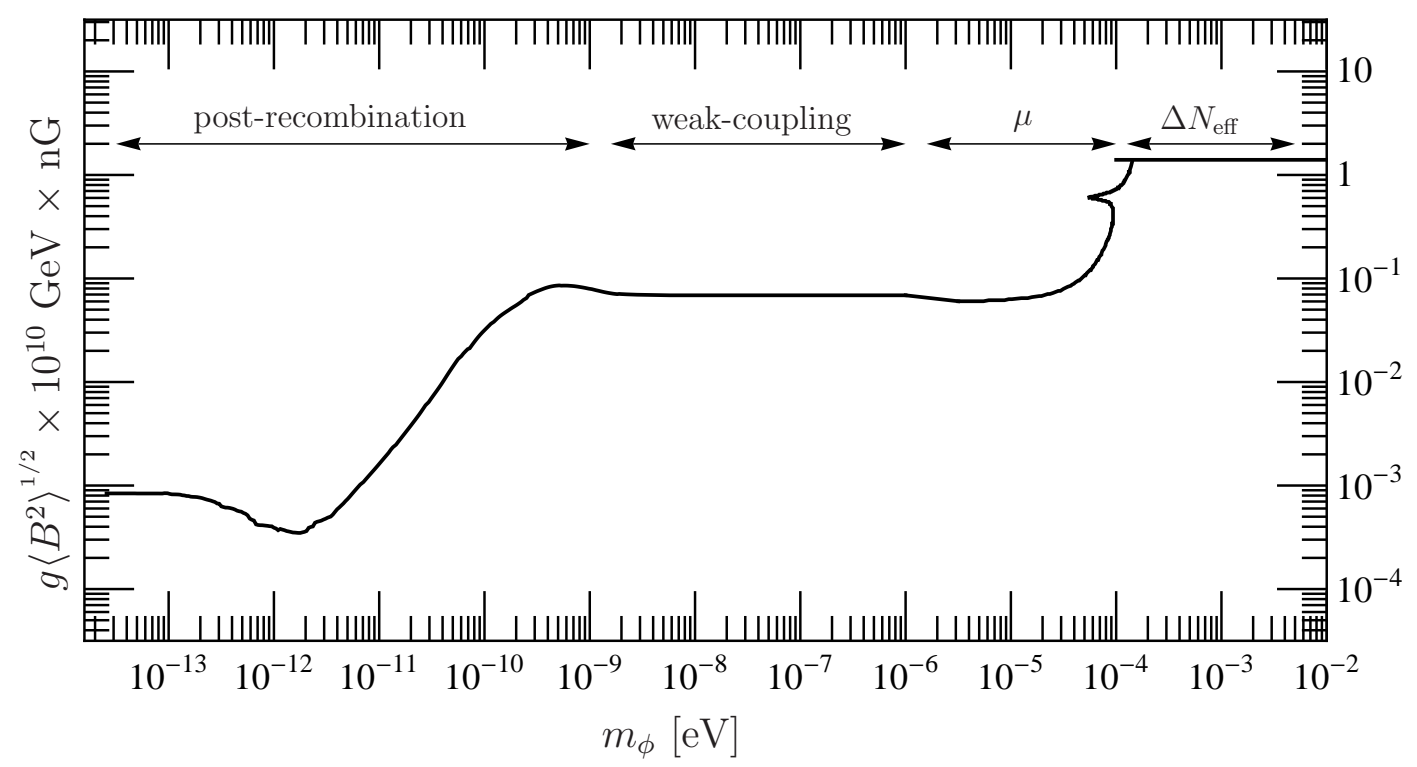

Figure 3. Bounds on the ALP parameter space from distortions of the CMB blackbody spectrum caused by resonant $\gamma \rightarrow \phi$ oscillations. The ALP mass determines the time of the resonant transition. In the region labeled as "post-recombination" the transition happens in the post-recombination epoch whereas outside this region the transition occurs before recombination and CMB last scattering. However, in the region labeled as "weak-coupling" the transitions happen when the plasma is so weakly coupled that it cannot process the distortions. In the region labeled $\mu$, Compton scattering would restore a Bose-Einstein spectrum with a chemical potential that can be erased through inverse double Compton scattering and Bremsstrahlung. For $m_{\phi} \gtrsim 0.1 \mathrm{meV}$ all distortions are erased but the produced ALPs still contribute to the cosmic radiation density and can be constrained by comparing the number of effective number of relativistic species at BBN and CMB decoupling. Here $g$ is the ALP-photon coupling constant and $\left\langle B^{2}\right\rangle^{1 / 2}$ an sky average of the comoving magnetic field during the resonance.

\section{Discussion and conclusions}

In this paper, we have calculated bounds on photon-ALP oscillations in the primordial magnetic field, deriving updated constraints from the high precision CMB spectrum data collected by the FIRAS instrument on board of COBE. A previous study [6] was derived in the pre-COBE era and it lacked a detailed treatment of the effects of the plasma medium on the photon-ALPs oscillations. This has motivated us to re-evaluate the bounds. We obtain limits on the product of the ALP-photon coupling $g$ times the sky and polarization averaged magnetic field $\left\langle B^{2}\right\rangle^{1 / 2}$,

$$
g\left\langle B^{2}\right\rangle^{1 / 2} \lesssim 10^{-13} \sim 10^{-11} \mathrm{GeV}^{-1} \mathrm{nG},
$$

for ALP masses between $10^{-14} \mathrm{eV}$ and $10^{-4} \mathrm{eV}$. Slightly weaker bounds were also derived for higher ALP masses.

Our bound nicely connects with the one obtained in [11] for ALP masses less than $10^{-14} \mathrm{eV}$, considering only non-resonant conversions today. Our argument allows to 
extend the sensitivity of CMB measurements also in a region of the parameter space that was previously unconstrained. We mention that ALPs can also be thermally produced in Early Universe via processes like $e \gamma \rightarrow e \phi$, forming a relic background. The $\phi \gamma \gamma$ vertex would also allow for radiative decays of these particles. In this case, additional cosmological bounds can be obtained for $m_{\phi} \gtrsim \mathrm{eV}$, as studied in [31].

There are several interesting proposals to measure the CMB spectrum with higher sensitivity than the FIRAS instrument. The DIMES (Diffuse Microwave Emission Survey) proposal [32] aims to probe the region of smaller frequencies $(2-100 \mathrm{GHz}$ corresponding to $0.035 \leq \omega_{0} / T_{0} \leq 1.8$ ) with sensitivity comparable to FIRAS. Unfortunately, as discussed above, the distortions grow with increasing frequency, so DIMES would not significantly strengthen bounds on axion-photon oscillations, in contrast to constraints on hidden photon - photon mixing for which conversion probabilities are inversely proportional to the photon energy. For this reason, the proposed FIRAS II [33] would be more relevant for photon-axion mixing constraints since it would shrink the FIRAS error bars by almost two order of magnitude and reach much higher frequencies $\left(60-3600 \mathrm{GHz}\right.$, or $\left.1 \leq \omega_{0} / T_{0} \leq 65\right)$. If such a project is realised the sensitivity to photon-ALP oscillations will very likely improve by more than an order of magnitude in $g\left\langle B^{2}\right\rangle^{1 / 2}$. A slight improvement on our bound in the pre-recombination era could be achieved thanks to new experiments, like ARCADE (Absolute Radiometer for Cosmology, Astrophysics, and Diffuse Emission) which would constrain the chemical potential of the CMB spectrum $|\mu|$ down to $2 \times 10^{-5}$ [34].

Let us recall that recent results from the CAST experiment [35-37] give a direct experimental bound on the ALP-photon coupling of $g \lesssim 8.8 \times 10^{-11} \mathrm{GeV}^{-1}$ for $m_{\phi} \lesssim 0.02 \mathrm{eV}$, slightly stronger than the long-standing globular-cluster limit [38]. For ultra-light ALPs $\left(m_{\phi} \lesssim 10^{-10} \mathrm{eV}\right)$ a stringent limit from the absence of $\gamma$-rays from SN 1987A gives $g \lesssim 1 \times 10^{-11} \mathrm{GeV}^{-1}$ [39] or even $g \lesssim 3 \times 10^{-12} \mathrm{GeV}^{-1}$ [40]. We stress that without direct evidence for a primordial magnetic field, our bounds on $g\left\langle B^{2}\right\rangle^{1 / 2}$ do not allow to constrain directly the coupling constant $g$. However, if a primordial magnetic field would be found with values close to the current upper bound, the resulting CMB limit on $g$ for $m_{\phi} \lesssim 10^{-4} \mathrm{eV}$ would overcome the barrier placed by current experimental and astrophysical bounds. Conversely, if ALPs will be eventually discovered improving the current sensitivity of the solar axion helioscope CAST, or with new techniques [4143] in laser experiments like photon regeneration [44-47] or laser polarization [43, 48], our cosmological argument will provide a complementary constrain on the strength of the primordial magnetic field.

Moreover, we note that our limit applies also to the so called chameleon-like scalar particles mixing with photons $[49,50]$. Such particles are introduced to explain the acceleration of the Universe, as inflation or dark energy fields [51], or to cause variations in the fundamental constants $[52,53]$. In general, chameleons have properties that depend on the environment. For this reason, they can evade astrophysical and CAST bounds, since in the dense stellar environment they become so heavy that they can not be produced in the usual reactions [18]. In these cases one has thus to rely on 
the limits from the laboratory experiments, which take place essentially in vacuum. For a wide class of chameleon models, the laser PVLAS experiment would rule out values of $g \gtrsim 5 \times 10^{-7} \mathrm{GeV}^{-1}$ [54] (see also [55-58]). Strong bounds also come from observations of starlight polarization [59]. In the expanding Universe, given the low plasma density, these particles would behave essentially as standard ALPs, so that our bounds can be directly applied to this case. In this sense, if primordial magnetic fields will be discovered, our argument would rule-out the recently proposed mechanism of chameleon-photon conversions to explain the observed supernovae Ia brightening [60].

Furthermore, let us point that the kind of analysis performed in this work can be also extended to other particles having two-photon vertices and sub-eV masses. For instance, a very similar analysis could be performed to massive spin-2 particles [61] like Kaluza-Klein gravitons [62].

As a final remark, our study is relevant for recent studies of conversions of highenergy gamma rays into ALPs in the intergalactic magnetic field in relation to the observed cosmic transparency of high energy gamma sources [63-65] or to ultra highenergy cosmic ray propagation [66]. If eventually a primordial magnetic field will be discovered, a definitive verdict on the impact of these fascinating mechanisms would require a combined analysis including our new CMB constraints. In this sense, it is nice to realize that our cosmological limits obtained with microwave photons could have relevant consequences for signatures of ALPs in high-energy gamma sources. This confirms once more the broad range of energies potentially accessible through axion-like particle searches.

\section{Acknowledgements}

This work was supported by the Deutsche Forschungsgemeinschaft (SFB 676 "Particles, Strings and the Early Universe: The Structure of Matter and Space-Time) and by the European Union ILIAS (contract No. RII3-CT-2004-506222). The work of A.M. is

supported by the Italian Istituto Nazionale di Fisica Nucleare (INFN). We thank Georg Raffelt and Andreas Ringwald for reading the manuscript and for valuable comments on it. A.M. acknowledges kind hospitality at the Hamburg University during the development of this work.

\section{References}

[1] P. P. Kronberg, "Extragalactic magnetic fields," Rept. Prog. Phys. 57, 325 (1994).

[2] D. Grasso and H. R. Rubinstein, "Magnetic fields in the early universe," Phys. Rept. 348, 163 (2001) [astro-ph/0009061].

[3] WMAP Collaboration, G. Hinshaw et al., "Five-Year Wilkinson Microwave Anisotropy Probe Observations:Data Processing, Sky Maps, \& Basic Results," Astrophys. J. Suppl. 180, 225 (2009) [arXiv:0803.0732 [astro-ph]].

[4] P. Blasi, S. Burles and A. V. Olinto, "Cosmological Magnetic Fields Limits in an Inhomogeneous Universe," Astrophys. J. 514, L79 (1999) [astro-ph/9812487]. 
[5] T. Kahniashvili, Y. Maravin and A. Kosowsky, "Primordial Magnetic Field Limits from WMAP Five-Year Data," arXiv:0806.1876 [astro-ph].

[6] T. Yanagida and M. Yoshimura, "Resonant axion-photon conversion in the Early Universe," Phys. Lett. B 202, 301 (1988).

[7] D. J. Fixsen, E. S. Cheng, J. M. Gales, J. C. Mather, R. A. Shafer and E. L. Wright, "The Cosmic Microwave Background Spectrum from the Full COBE/FIRAS Data Set," Astrophys. J. 473, 576 (1996) [astro-ph/9605054].

[8] J. C. Mather, D. J. Fixsen, R. A. Shafer, C. Mosier and D. T. Wilkinson, "Calibrator Design for the COBE Far Infrared Absolute Spectrophotometer (FIRAS)," Astrophys. J. 512, 511 (1999) [astro-ph/9810373].

[9] J. Jaeckel, J. Redondo and A. Ringwald, "Signatures of a hidden cosmic microwave background," Phys. Rev. Lett. 101, 131801 (2008) [arXiv:0804.4157 [astro-ph]].

[10] A. Mirizzi, J. Redondo and G. Sigl, "Microwave Background Constraints on Mixing of Photons with Hidden Photons," JCAP 0903, 026 (2009) [arXiv:0901.0014 [hep-ph]].

[11] A. Mirizzi, G. G. Raffelt and P. D. Serpico, "Photon axion conversion as a mechanism for supernova dimming: Limits from CMB spectral distortion," Phys. Rev. D 72, 023501 (2005) [astro$\mathrm{ph} / 0506078]$.

[12] A. Mirizzi, D. Montanino and P. D. Serpico, "Revisiting cosmological bounds on radiative neutrino lifetime," Phys. Rev. D 76, 053007 (2007) [arXiv:0705.4667 [hep-ph]].

[13] A. Melchiorri, A. Polosa and A. Strumia, "New bounds on millicharged particles from cosmology," Phys. Lett. B 650, 416 (2007) [hep-ph/0703144].

[14] G. Raffelt, "Stars as laboratories for fundamental physics," The University of Chicago Press (1996).

[15] E. Masso and J. Redondo, "Evading Astrophysical Constraints on Axion-Like Particles," JCAP 0509, 015 (2005) [hep-ph/0504202].

[16] J. Jaeckel, E. Masso, J. Redondo, A. Ringwald and F. Takahashi, "The Need for Purely LaboratoryBased Axion-Like Particle Searches," Phys. Rev. D 75, 013004 (2007) [hep-ph/0610203].

[17] E. Masso and J. Redondo, "Compatibility of CAST search with axion-like interpretation of PVLAS results," Phys. Rev. Lett. 97, 151802 (2006) [hep-ph/0606163].

[18] P. Brax, C. van de Bruck and A. C. Davis, "Compatibility of the chameleon-field model with fifthforce experiments, cosmology, and PVLAS and CAST results," Phys. Rev. Lett. 99, 121103 (2007) [hep-ph/0703243].

[19] R. N. Mohapatra and S. Nasri, Phys. Rev. Lett. 98, 050402 (2007) [arXiv:hep-ph/0610068].

[20] E. Masso and R. Toldra, "On a Light Spinless Particle Coupled to Photons," Phys. Rev. D 52, 1755 (1995) [hep-ph/9503293].

[21] G. Raffelt and L. Stodolsky, "Mixing of the Photon with Low Mass Particles," Phys. Rev. D 37, 1237 (1988).

[22] L. Wolfenstein, "Neutrino oscillations in matter," Phys. Rev. D 17, 2369 (1978).

[23] S. P. Mikheyev and A. Y. Smirnov, "Resonance enhancement of oscillations in matter and solar neutrino spectroscopy," Yad. Fiz. (1985) no. 42, 1441. [Sov. J. Nucl. Phys. 42, 913 (1985)].

[24] S. P. Mikheyev and A. Y. Smirnov, "Resonant amplification of neutrino oscillations in matter and solar neutrino spectroscopy," Nuovo Cimento C (1986) no. 9, 17.

[25] S. J. Parke, "Nonadiabatic Level Crossing in Resonant Neutrino Oscillations," Phys. Rev. Lett. 57, 1275 (1986).

[26] A. B. Balantekin, S. H. Fricke and P. J. Hatchell, "Analytical And Semiclassical Aspects Of Matter Enhanced Neutrino Oscillations," Phys. Rev. D 38, 935 (1988).

[27] S. Seager, D. D. Sasselov, and D. Scott, "A New Calculation of the Recombination Epoch," astro$\mathrm{ph} / 9909275$.

[28] A. Mack, T. Kahniashvili and A. Kosowsky, "Vector and Tensor Microwave Background Signatures of a Primordial Stochastic Magnetic Field," Phys. Rev. D 65, 123004 (2002) [astro-ph/0105504].

[29] W. Hu and J. Silk, "Thermalization and spectral distortions of the cosmic background radiation," 
Phys. Rev. D 48, 485 (1993).

[30] F. Iocco, G. Mangano, G. Miele, O. Pisanti and P. D. Serpico, "Primordial Nucleosynthesis: from precision cosmology to fundamental physics," Phys. Rept. 472, 1 (2009) [arXiv:0809.0631 [astro$\mathrm{ph}]]$.

[31] E. Masso and R. Toldra, "New constraints on a light spinless particle coupled to photons," Phys. Rev. D 55, 7967 (1997) [hep-ph/9702275].

[32] A. Kogut, "Diffuse Microwave Emission Survey," astro-ph/9607100.

[33] D. J. Fixsen, and J. C. Mather, "The Spectral Results of the Far-Infrared Absolute Spectrophotometer Instrument on COBE," ApJ 581 817-822.

[34] J. Singal, "The CMB and galactic microwave absolute spectrum: Science and measurement with ARCADE 2," Mod. Phys. Lett. A 23, 1719 (2008).

[35] CAST Collaboration, S. Andriamonje et al., "An improved limit on the axion-photon coupling from the CAST experiment," JCAP 0704, 010 (2007) [hep-ex/0702006].

[36] CAST Collaboration, K. Zioutas et al., "First results from the CERN Axion Solar Telescope (CAST)," Phys. Rev. Lett. 94, 121301 (2005) [hep-ex/0411033].

[37] CAST Collaboration, E. Arik et al., "Probing eV-scale axions with CAST," JCAP 0902, 008 (2009) [arXiv:0810.4482 [hep-ex]].

[38] G. G. Raffelt, "Astrophysical axion bounds," Lect. Notes Phys. 741, 51 (2008) [hep-ph/0611350].

[39] J. W. Brockway, E. D. Carlson and G. G. Raffelt, "SN 1987A gamma-ray limits on the conversion of pseudoscalars," Phys. Lett. B 383, 439 (1996) [astro-ph/ 9605197].

[40] J. A. Grifols, E. Massó and R. Toldrà, "Gamma rays from SN 1987A due to pseudoscalar conversion," Phys. Rev. Lett. 77, 2372 (1996) [astro-ph/9606028].

[41] F. Hoogeveen and T. Ziegenhagen, "Production and detection of light bosons using optical resonators," Nucl. Phys. B 358, 3 (1991).

[42] P. Sikivie, D. B. Tanner and K. van Bibber, "Resonantly enhanced axion - photon regeneration," Phys. Rev. Lett. 98, 172002 (2007) [hep-ph/0701198].

[43] G. Cantatore, R. Cimino, M. Karuza, V. Lozza and G. Raiteri, "Polarization measurements and their perspectives: PVLAS Phase II," arXiv:0809.4208 [hep-ex].

[44] K. Ehret et al., "Resonant laser power build-up in ALPS - a light-shining-through-walls experiment -", arXiv:0905.4159 [physics.ins-det].

[45] BMV Collaboration, M. Fouche et al., "Search for photon oscillations into massive particles," Phys. Rev. D 78, 032013 (2008) [arXiv:0808.2800 [hep-ex]].

[46] GammeV Collaboration, A. S. Chou et al., "Search for axion-like particles using a variable baseline photon regeneration technique," Phys. Rev. Lett. 100, 080402 (2008) [arXiv:0710.3783 [hep-ex]].

[47] LIPSS Collaboration, A. Afanasev et al., "New Experimental limit on Optical Photon Coupling to Neutral, Scalar Bosons," Phys. Rev. Lett. 101, 120401 (2008) [arXiv:0806.2631 [hep-ex]].

[48] R. Battesti et al., "The BMV experiment : a novel apparatus to study the propagation of light in a transverse magnetic field," Eur. Phys. Journ. D46, Issue 2, pp.323-333 (2008), arXiv:0710.1703v1 [physics.optics].

[49] J. Khoury and A. Weltman, "Chameleon cosmology," Phys. Rev. D 69, 044026 (2004) [astro$\mathrm{ph} / 0309411]$.

[50] D. F. Mota and D. J. Shaw, "Strongly coupled chameleon fields: New horizons in scalar field theory," Phys. Rev. Lett. 97, 151102 (2006) [hep-ph/0606204].

[51] P. Brax, C. van de Bruck, A. C. Davis, J. Khoury and A. Weltman, "Detecting dark energy in orbit: The cosmological chameleon," Phys. Rev. D 70, 123518 (2004) [arXiv:astro-ph/0408415].

[52] D. F. Mota and J. D. Barrow, "Local and Global Variations of The Fine Structure Constant," Mon. Not. Roy. Astron. Soc. 349, 291 (2004) [arXiv:astro-ph/0309273].

[53] K. A. Olive and M. Pospelov, "Environmental Dependence of Masses and Coupling Constants," Phys. Rev. D 77, 043524 (2008) [arXiv:0709.3825 [hep-ph]].

[54] PVLAS Collaboration, E. Zavattini et al., "New PVLAS results and limits on magnetically 
Constraining resonant photon-axion conversions in the Early Universe

induced optical rotation and ellipticity in vacuum," Phys. Rev. D 77, 032006 (2008) [arXiv:0706.3419 [hep-ex]].

[55] M. Ahlers, A. Lindner, A. Ringwald, L. Schrempp and C. Weniger, "Alpenglow - A Signature for Chameleons in Axion-Like Particle Search Experiments," Phys. Rev. D 77, 015018 (2008) [arXiv:0710.1555 [hep-ph]].

[56] P. Brax, C. van de Bruck, A. C. Davis, D. F. Mota and D. J. Shaw, "Testing Chameleon Theories with Light Propagating through a Magnetic Field," Phys. Rev. D 76, 085010 (2007) [arXiv:0707.2801 [hep-ph]].

[57] H. Gies, D. F. Mota and D. J. Shaw, "Hidden in the Light: Magnetically Induced Afterglow from Trapped Chameleon Fields," Phys. Rev. D 77, 025016 (2008) [arXiv:0710.1556 [hep-ph]].

[58] GammeV Collaboration, A. S. Chou et al., "A Search for chameleon particles using a photon regeneration technique," Phys. Rev. Lett. 102, 030402 (2009) [arXiv:0806.2438 [hep-ex]].

[59] C. Burrage, A. C. Davis and D. J. Shaw, "Detecting Chameleons: The Astronomical Polarization Produced by Chameleon-like Scalar Fields," Phys. Rev. D 79, 044028 (2009) [arXiv:0809.1763 [astro-ph]].

[60] C. Burrage, "Supernova Brightening from Chameleon-Photon Mixing," Phys. Rev. D 77, 043009 (2008) [arXiv:0711.2966 [astro-ph]].

[61] C. Biggio, E. Masso and J. Redondo, "Mixing of photons with massive spin-two particles in a magnetic field," Phys. Rev. D 79, 015012 (2009) [hep-ph/0604062].

[62] C. Deffayet and J. P. Uzan, "Photon mixing in universes with large extra-dimensions," Phys. Rev. D 62, 063507 (2000) [hep-ph/0002129].

[63] A. De Angelis, O. Mansutti and M. Roncadelli, "Axion-Like Particles, Cosmic Magnetic Fields and Gamma-Ray Astrophysics," Phys. Lett. B 659, 847 (2008) [arXiv:0707.2695 [astro-ph]].

[64] A. De Angelis, O. Mansutti and M. Roncadelli, "Evidence for a new light spin-zero boson from cosmological gamma-ray propagation?," Phys. Rev. D 76, 121301 (2007) [arXiv:0707.4312 [astro$\mathrm{ph}]$.

[65] A. De Angelis, O. Mansutti, M. Persic and M. Roncadelli, "Photon propagation and the VHE gamma-ray spectra of blazars: how transparent is really the Universe?," arXiv:0807.4246 [astro$\mathrm{ph}]$.

[66] C. Csaki, N. Kaloper, M. Peloso and J. Terning, "Super-GZK photons from photon axion mixing," JCAP 0305, 005 (2003) [hep-ph/0302030]. 